\title{
Antimicrobial resistance in Neisseria gonorrhoeae and Mycoplasma genitalium isolates from the private healthcare sector in South Africa: A pilot study
}

\author{
L D Maduna, ${ }^{1,2}$ PhD; R P H Peters, ${ }^{1,3,4} \mathrm{PhD}$; C Kingsburgh, ${ }^{5} \mathrm{MD}$; K-A Strydom, ${ }^{5} \mathrm{MD} ; \mathrm{M}$ M Kock, ${ }^{1,6} \mathrm{PhD}$ \\ ${ }^{1}$ Department of Medical Microbiology, Faculty of Health Sciences, University of Pretoria, South Africa \\ ${ }^{2}$ Department of Biology, Faculty of Science and Technology, National University of Lesotho, Maseru, Lesotho \\ ${ }^{3}$ Department of Medical Microbiology, CAPHRI School of Public Health and Primary Care, Maastricht University Medical Centre, Maastricht, \\ The Netherlands \\ ${ }^{4}$ Division of Medical Microbiology, Faculty of Health Sciences, University of Cape Town, South Africa \\ ${ }^{5}$ Ampath National Reference Laboratory, Pretoria, South Africa \\ ${ }^{6}$ Tshwane Academic Division, National Health Laboratory Service, Pretoria, South Africa
}

Corresponding author: L D Maduna (ld.maduna@nul.ls)

Background. Reports have emerged globally of antimicrobial resistance (AMR) in Neisseria gonorrhoeae and Mycoplasma genitalium infections. In South Africa (SA), there are substantial differences between private and public healthcare with regard to antimicrobial drug prescribing practice, which could affect AMR patterns of private and public healthcare patients.

Objectives. To perform a pilot study to determine the frequency of AMR of $N$. gonorrhoeae and M. genitalium in patients accessing SA's private healthcare sector.

Methods. In this cross-sectional study, N. gonorrhoeae-positive cultures and M. genitalium DNA samples were collected from a private healthcare reference laboratory from August 2018 to August 2019. In N. gonorrhoeae-positive cultures, antimicrobial susceptibility testing was performed, followed by $N$. gonorrhoeae multiantigen sequence typing (NG-MAST) to determine genetic relatedness of the isolates. To determine macrolide and fluoroquinolone resistance rates, $M$. genitalium-positive samples were analysed by sequencing the $23 \mathrm{~S}$ rRNA, gyr $\mathrm{A}$ and parC genes.

Results. Twenty-one N. gonorrhoeae- and $27 \mathrm{M}$. genitalium-positive specimens were included in this analysis. High rates of resistance were detected among gonococcal isolates, with $90 \%$ resistance to tetracycline, $86 \%$ to penicillin and $62 \%$ to ciprofloxacin, but no resistance to azithromycin, cefixime and ceftriaxone. NG-MAST revealed genetically diverse isolates with $83 \%$ novel NG-MAST sequence types. Macrolide and fluoroquinolone resistance-associated mutations were detected in $18.5 \%(n=5 / 27)$ and $7.4 \%$ ( $n=2 / 27)$ of $M$. genitalium strains, respectively.

Conclusions. We observed high frequencies of ciprofloxacin, penicillin and tetracycline resistance in $N$. gonorrhoeae and macrolide resistance-associated mutations in $M$. genitalium in private healthcare sector patients in SA. This finding highlights the need to use diagnostics for sexually transmitted infections and to include the private healthcare sector in antimicrobial surveillance and stewardship programmes.

S Afr Med J 2021;111(10):995-997. https://doi.org/10.7196/SAMJ.2021.v111i10.15714

The increasing prevalence of antimicrobial resistance (AMR) in the sexually transmitted infections (STIs) Neisseria gonorrhoeae and Mycoplasma genitalium is a major concern worldwide. ${ }^{[1]}$ In South Africa (SA), STIs are treated syndromically based on the patient's presenting symptoms and using a combination of broadspectrum empirical antibiotics. The standard regimen for urethral and vaginal discharge syndrome is ceftriaxone and azithromycin with or without metronidazole. ${ }^{[2]}$ An important limitation of the syndromic management approach is that there are limited data on the occurrence of AMR in N. gonorrhoeae and M. genitalium, owing to the lack of routine laboratory diagnostics.

The healthcare system in SA is two-tiered: the public healthcare sector provides free healthcare and serves the majority of the population $(>80 \%)$, while private healthcare is accessed by the minority (15-17\%) and is largely paid for through private health insurance. ${ }^{[3]}$ Both sectors use syndromic treatment for STI management. ${ }^{[2]}$ However, most of the AMR data have been reported from the public healthcare sector. The national STI sentinel surveillance is conducted only in public sector facilities, and research studies have focused on that sector as well. ${ }^{[4-7]}$ Research on the occurrence of AMR in STIs in the private healthcare sector therefore remains limited. ${ }^{[8,9]}$

There are substantial differences between the private and public healthcare sectors with regard to patients' healthcareseeking behaviour, antimicrobial drug prescribing practice, and socioeconomic status of the patient population. ${ }^{[10]}$ These factors could all impact on the occurrence of antimicrobial-resistant STIs and result in different epidemiological profiles in the two sectors.

\section{Objectives}

To conduct a pilot study to determine the frequency of AMR of N. gonorrhoeae and M. genitalium in patients accessing SA's private healthcare sector.

\section{Methods}

In this cross-sectional study, N. gonorrhoeae-positive cultures and M. genitalium DNA samples with linked anonymous clinical 
information were collected from a private healthcare sector diagnostic reference laboratory in Pretoria, SA, from August 2018 to August 2019. This reference laboratory receives specimens for pathology services from all provinces in SA. The laboratory uses New York City (NYC) agar to culture and matrix-assisted laser desorption/ ionisation time-of-flight mass spectrometry (MALDI-TOF MS; Bruker, USA) to identify N. gonorrhoeae, and the Anyplex II STI-7 assay (Seegene, Korea) to detect M. genitalium. Cultured N. gonorrhoeae and M. genitalium DNA were transported to the Department of Medical Microbiology at the University of Pretoria for further resistance testing.

At the University of Pretoria, N. gonorrhoeae was confirmed by Gram stain, rapid oxidase test and API NH (bioMérieux, France). Minimum inhibitory concentration (MICs) were determined using E-tests (bioMérieux, France) on the GC-agar base (Oxoid, UK) for tetracycline, ciprofloxacin, penicillin G, azithromycin, ceftriaxone, cefixime and spectinomycin. MICs were interpreted according to the European Committee of Antimicrobial Susceptibility Testing breakpoints, except for azithromycin, where epidemiological cut-off (ECOFF) values were used because no resistance breakpoints exist (http://www. eucast.org). N. gonorrhoeae ATCC 49266 strain was used as quality control. Molecular typing of gonococcal isolates was done using the N. gonorrhoeae multiantigen sequence typing (NG-MAST) as previously described. ${ }^{[1]}$ Sequences were submitted to the NG-MAST database (http://www.ng-mast.net) for assignment of sequence types (STs).

The presence of $M$. genitalium DNA was confirmed using a validated real-time polymerase chain reaction (PCR) assay targeting the $\mathrm{MgPa}$ adhesin gene. ${ }^{[11]}$ Macrolide drug resistance-associated mutations in the 23S rRNA gene were identified using real-time PCR assay coupled with melting curve analysis for detection of point mutations. ${ }^{[12]}$ Samples with a temperature peak different from the wild-type strains were sequenced to confirm mutations in the $23 \mathrm{~S}$ rRNA gene. ${ }^{[13]}$ Quinolone resistance-associated mutations were detected by sequencing of quinolone resistance-determining regions (QRDRs) of gyrA and parC genes in macrolide-resistant strains, as previously described. ${ }^{[13]}$ Ethical approval was obtained from the Research Ethics Committee at the Faculty of Health Sciences, University of Pretoria (ref. nos 253/2017, 207/2018 and 171/2019).

\section{Results}

Twenty-one N. gonorrhoeae isolates were collected over the study period, 19 from urethral swabs and 2 from vaginal swabs. Gonococcal isolates showed high rates of resistance to tetracycline $(n=19 / 21$; $90 \%)$, penicillin ( $n=18 / 21 ; 86 \%)$ and ciprofloxacin $(n=13 / 21 ; 62 \%)$. All isolates were susceptible to the cephalosporins and spectinomycin; MICs for azithromycin were all below the ECOFF.

NG-MAST classified 21 isolates into 12 different STs; $10(n=10 / 12$; $83 \%)$ of these STs have not been described previously. Seven $(n=7 / 12)$
STs were identified as unrelated singletons. Frequent STs were ST3609 ( $n=3$ isolates), ST6216 $(n=2)$, a novel ST containing porB allele 1320 and $t b p \mathrm{~B}$ allele $623(n=4)$, and a novel ST containing por B allele 11132 and $t b p \mathrm{~B}$ allele $136(n=3)$.

M. genitalium was confirmed in 27/27 (100\%) of DNA samples received; 21 were from male urine samples, 4 from vaginal swabs and 2 from urethral swabs. Melting curve analysis of DNA identified five strains that had a different melting temperature $\left(62-64.7^{\circ} \mathrm{C}\right)$ from the wild-type strain $\left(69^{\circ} \mathrm{C}\right)$. Sequence analysis confirmed macrolide resistance-associated mutations in the $23 \mathrm{~S}$ rRNA in $18.5 \%(n=5 / 27)$ of M. genitalium strains; an A-to-G transition was detected at nucleotide position 2071 in 3 strains and at position 2072 in 2 (Table 1). Various amino acid alterations were observed in the ParC and GyrA of the QRDR; two strains had a Gln-132 $\rightarrow$ Arg substitution in the GyrA and the other one had a silent mutation (A318G). In addition, one strain had a Ser83 $\rightarrow$ Ile substitution in the ParC and a concurrent gry $A$ mutation $(\mathrm{Gln}-132 \rightarrow \operatorname{Arg}$ ) that has been associated with moxifloxacin and sitafloxacin treatment failures. ${ }^{[12]}$ One isolate had a novel Met$55 \rightarrow$ Leu substitution in the ParC of unknown clinical significance. Coexistent macrolide and quinolone resistance-associated mutations were detected in 2 patients.

\section{Discussion}

This pilot study reports on antimicrobial-resistant N. gonorrhoeae and M. genitalium infections in patients accessing the private healthcare sector in SA. We demonstrated high rates of AMR in N. gonorrhoeae isolates for some of the previously used antibiotics and concurrent macrolide and quinolone resistance-associated mutations in some of the M. genitalium strains. Although specimen collection was done over a 1-year period in a large reference laboratory, the number of strains obtained was limited, highlighting that diagnostic testing for STIs is performed only on a small scale in the private healthcare sector.

High frequencies of tetracycline and ciprofloxacin resistance were detected for gonococcal isolates; these drugs have been extensively used in the syndromic management of STIs in SA in the past two decades. ${ }^{[2]}$ These findings are consistent with national surveillance data and a recent study in KwaZulu-Natal Province showing high rates of resistance to these antimicrobials in the public sector. ${ }^{[7,4]} \mathrm{In}$ addition, a higher frequency of resistance to penicillin (89\%) was observed in gonococcal isolates in our study compared with the national sentinel surveillance, which reports a penicillin resistance prevalence of $51 \%{ }^{[4]}$ This finding may be explained by a higher consumption of amoxicillin and amoxicillin/clavulanic acid in the private healthcare sector for other unrelated infections such as respiratory tract infections. ${ }^{[14]}$ We did not observe azithromycin resistance in this selected group of gonococcal isolates from the

Table I. Macrolide resistance-associated mutations and fluoroquinolone resistance-associated amino acid changes in GyrA and ParC in Mycoplasma genitalium from private healthcare sector patients in South Africa

\begin{tabular}{|c|c|c|c|c|c|c|}
\hline \multirow[b]{2}{*}{ Patient no. } & \multirow[b]{2}{*}{ Province } & \multirow[b]{2}{*}{ Gender } & \multirow[b]{2}{*}{ Specimen type } & \multirow[b]{2}{*}{ 23SrRNA ${ }^{*}$ mutation } & \multicolumn{2}{|c|}{ Amino acid change ${ }^{*}$} \\
\hline & & & & & GyrA & ParC \\
\hline 1 & Western Cape & $\mathrm{M}$ & Urine & A2071G & Wild type & Wild type \\
\hline 2 & Western Cape & M & Urine & A2071G & Gln- $132 \rightarrow \operatorname{Arg}$ & Met- $55 \rightarrow$ Leu \\
\hline 3 & Gauteng & M & Urine & A2072G & Wild type & Wild type \\
\hline 4 & Gauteng & M & Urethral swab & A2072G & Silent mutation $^{\dagger}$ & Wild type \\
\hline 5 & Gauteng & M & Urethral swab & A2071G & Gln-132 $\rightarrow$ Arg & Ser83 $\rightarrow$ Ile \\
\hline
\end{tabular}


private healthcare sector, which is somewhat unexpected as there are concerns about emerging azithromycin resistance in the public healthcare sector. ${ }^{[7]}$ Molecular epidemiological analysis of the gonococcal isolates showed genetic diversity, with a high number of novel NG-MAST STs that have not been described elsewhere.

A high frequency of macrolide resistance-associated mutations was detected in M. genitalium strains; this is in stark contrast to data from the public sector, where a low prevalence of macrolide resistance-associated mutations has been reported in several studies. ${ }^{[5,6,15]}$ The observed high rate of macrolide resistance in the private healthcare sector could be due to selection bias, i.e. patients with urethritis not responding to empirical treatment may be more likely to have a diagnostic test. It could also result from different prescribing behaviour; azithromycin is commonly used for treatment of respiratory infections in the private healthcare sector, but not in the public sector. ${ }^{[14]}$ Of concern is that we detected M. genitalium strains with coexistent macrolide and quinolone resistance-associated mutations in two patients.

\section{Study limitations}

This pilot study has several limitations. First, the relatively small sample size and geographical spread may not be fully representative of the population in the private sector; however, it does give an important insight into the situation and highlights the need for further research. Second, there was probably selection bias of patients who had specimens sent for laboratory diagnostics, as these are expensive, patients can generally not afford such tests, and syndromic treatment remains the cornerstone of STI management. Lastly, the therapeutic outcomes of the patients with dual-class resistance in M. genitalium associated with treatment failures were not available owing to the anonymised study design.

\section{Conclusions}

High frequencies of AMR in N. gonorrhoeae and macrolide resistance in M. genitalium were detected from patients in the private healthcare sector. This study highlights the importance of introducing routine STI diagnostics and inclusion of the private sector in AMR surveillance programmes.
Author contributions. LDM, RPHP and MMK designed, initiated and co-ordinated the study. LDM, CK and K-AS performed all the laboratory analyses. CK and K-AS supplied patient data. LDM, RPHP and MMK analysed and interpreted all the data, and wrote a first draft of the manuscript. LDM, RPHP, CK, K-AS and MMK read, commented on and approved the final manuscript.

Funding. This work was supported by a grant received from the National Health Laboratory Service Research Trust (GRANT00494646).

Conflicts of interest. None.

1. Unemo M, Jensen JS. Antimicrobial-resistant sexually transmitted infections: gonorrhoea and Mycoplasma genitalium. Nat Rev Urol 2017;14(3):139-152 https://doi.org/10.1038/nrurol.2016.268 National Department of Health, South Africa. Sexually transmitted infections: Management guidelines 2015. http://www.kznhealth.gov.za/family/STI-guidelines-2015.pdf (accessed 20 January 2021).

. Statistics South Africa. General household survey 2018. Statistical release P0318. http://www.statssa. gov.za/publications/P0318/P03182018.pdf (accessed 12 May 2020)

4. Kularatne R, Maseko V, Gumede L, Kufa T. Trends in Neisseria gonorrhoeae antimicrobial resistance over a ten-year surveillance period, Johannesburg, South Africa, 2008 - 2017. Antibiotics 2018;7(3):58. https://doi.org/10.3390/antibiotics7030058

5. Maduna LD, Laumen JG, Radebe O, Kock MM, Peters RP. Failure of syndromic management due to drug-resistant Mycoplasma genitalium infection in South Africa: A case report. Int J STD AIDS to drug-resistant Mycoplasma genitalium infection in South Africicis
2019;30(5):519-521. https://doi.org/10.1177/0956462418820745

6. Laumen JGE, van Alphen LB, Maduna LD, et al. Molecular epidemiological analysis of Mycoplasma genitalium shows low prevalence of azithromycin resistance and a well-established epidemic in South Africa. Sex Transm Infect 2021;97:152-156. https://doi.org/10.1136/sextrans-2019-054371

7. Rambaran S, Naidoo K, Dookie N, Moodley P, Sturm AW. Resistance profile of Neisseria gonorrhoeae in KwaZulu-Natal, South Africa questioning the effect of the currently advocated dual therapy. Sex Transm Dis 2019;46(4):266-270. https://doi.org/10.3390/antibiotics7030058

8. De Jongh M, Dangor Y, Adam A, Hoosen AA. Gonococcal resistance: Evolving from penicillin, tetracycline to the quinolones in South Africa - implications for treatment guidelines. Int J STD AIDS 2007;18(10):697-699. https://doi.org/10.1258/095646207782193768

9. Lewis D, Sriruttan C, Coetzee J. Detection of multidrug-resistant gonorrhoea in the Gauteng province. S Afr J Infect 2012;27(4):199-200. https://doi.org/10.1080/10158782.2012.11441509

10. Schneider H, Blaauw D, Dartnall E, Coetzee D, Ballard R. STD care in the South African private health sector. S Afr Med J 2001;91(2):151-156.

11. Martin IM, Ison CA, Aanensen DM, Fenton KA, Spratt BG. Rapid sequence-based identification of gonococcal transmission clusters in a large metropolitan area. J Infect Dis 2004;189(8):1497-505. gonococcal transmission clust
https://doi.org/10.1086/383047

12. Edberg A, Jurstrand M, Johansson E, et al. A comparative study of three different PCR assays for detection of Mycoplasma genitalium in urogenital specimens from men and women. J Med Microbio 2008:57(3):304-309. https://doi.org/10.1099/jmm.0.47498-0

13. Xiao L, Waites KB, van der Pol B, Aaron KJ, Hook EWI, Geisler WM. Mycoplasma genitalium infections with macrolide and fluoroquinolone resistance-associated mutations in heterosexual African American couples in Alabama. Sex Transm Dis 2019;46(1):18-24. https://doi.org/10.1097/ OLQ.0000000000000891

14. Schellack N, Benjamin D, Brink A, et al. A situational analysis of current antimicrobial governance, regulation, and utilisation in South Africa. Int J Infect Dis 2017;64:100-106. https://doi.org/10.1016/j. ijid. 2017.09 .002

15. Müller EE, Mahlangu MP, Lewis DA, Kularatne RS. Macrolide and fluoroquinolone resistanceassociated mutations in Mycoplasma genitalium in Johannesburg, South Africa, 2007 - 2014. BMC Infect Dis 2019;19(1):148. https://doi.org/10.1186/s12879-019-3797-6
Declaration. None.

Acknowledgements. None 